\title{
The SuperK-gadolinium project
}

\author{
Luis Labarga ${ }^{* i}$ \\ University Autonoma Madrid \\ E-mail: luis.labarga@uam.es \\ on behalf of The Super-Kamiokande Collaboration
}

The Super-Kamiokande (SK) Collaboration has committed to the SuperK-Gadolinium project that, by dissolving a Gd salt at $0.2 \%$ in mass in the SK water, will upgrade the detector to be able to identify neutrons with very high efficiency. The refurbishment of SK and the beginning of this new phase will start in June 2018. In this talk we present the physics benefits of high efficiency neutron tagging in Super-Kamiokande, the very extensive R\&D program followed towards the decision, and the most relevant steps in the implementation of the SuperK-Gd project.

The European Physical Society Conference on High Energy Physics

5-12 July, 2017

Venice

*Speaker.

${ }^{\dagger}$ supported by EU H2020-MSCA-RISE-2014-GA641540, SKPLUS. 


\section{Introduction}

The Super-Kamiokande experiment is currently the world's most powerful scientific apparatus for nucleon decay and $v$ physics and astrophysics $[1,2,3,4]$. From an instrumental point of view, the success of SK comes from the water-Cherenkov technique, that allows to instrument huge amounts of active mass with a relatively simple, reliable, understood and low cost technology [6].

However it has drawbacks, f.i. an inability to distinguish $v_{\mathrm{s}}$ from $\bar{v} \mathrm{~s}$. In this context Beacon and Vagins proposed [7] the addition to the SK water of a Gadolinium solute. The Gd has a very large cross-section for thermal neutron capture that is accompanied by the release of energetic $\gamma \mathrm{s}$, thus allowing SK a very efficient $(\approx 90 \%$ capture, $\approx 90 \%$ reconstruction) tagging of neutrons. This will open to Super-Kamiokande a whelm of processes of major scientific interest (Section 2). The SK Collaboration undertook a successful R\& D program (named originally GADZOOKS!) in this direction. The "proof-of-principle" was published in [8]. The key elements and conclusions of the $\mathrm{R} \& \mathrm{D}$ are presented in section 3 . As a result: we will add $\approx 100$ tons of the water-soluble gadolinium compound $\mathrm{Gd}_{2}\left(\mathrm{SO}_{4}\right)_{3}$ to the SK ultra-pure water. In Section 4 we describe the main challenges of the project and its present status.

\section{Physics Benefits}

Very high efficiency neutron tagging will be the key for the expected discovery by SuperK-Gd of the so-called Supernova Relic Neutrino (SRN). Fig. 1-left shows the energy dependence of the measured flux of low energy electron $v$ s by SK along with a sample of reasonable predictions for the corresponding spectrum of SRN. The background (mainly from spallation) and the solar flux itself, is above the SRN expectation by many orders of magnitude. The ability of unambiguously identify inverse $\beta$ reactions of $\bar{v}$ on proton $\left(\bar{v}_{e} p \rightarrow e^{+} n\right)$ from the coincidence in time (within tens of $\mu \mathrm{s}$ ) and space (within tens of $\mathrm{cm}$ ) between the Cherenkov lights of the $e^{+}$and the $e^{-}$,s Comptonscattered from the $\gamma$, s released by the Gd (Fig. 1-middle), allows SK-Gd to reduce dramatically the background to levels already similar to current expectations for SRN [5] (Fig. 1-right).
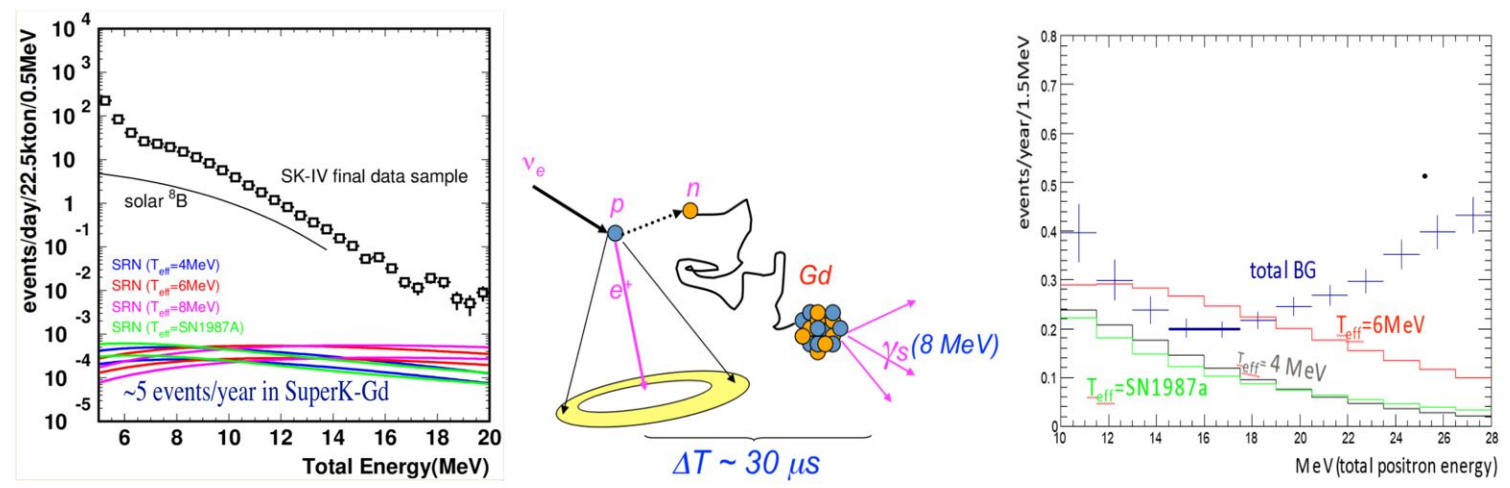

Figure 1: Supernova Relic Neutrino with SuperK-Gd (see text for details)

To have $\mathrm{Gd}_{2}\left(\mathrm{SO}_{4}\right)_{3}$ in SK will be most beneficial in the study of those fundamental processes that produce $\bar{v}$ signal in the water from inverse $\beta$ events. Remarkable is the much better characterization of a nearby supernova. Further, if the exploding star is big and rather close $(\sim 1 \mathrm{kpc}$ or 
less), we could get an early warning of its impending collapse [9] from the sudden and continuing increase of our usual SLE singles rate. This will be originated by the Gd-captured neutrons produced by the $\bar{v}_{\mathrm{s}}$ just above inverse $\beta$ threshold that are created in the $e^{+} e^{-}$annihilations within the volume of the raising-temperature star. On the other hand inverse $\beta$ events will open SK to a high efficiency detection of long-distance $\bar{v}$ s from reactors (if available): ten years of running will significantly improve the world's uncertainty of all accessible oscillation parameters.

At higher energies (order of $\mathrm{GeV}$ ) $\bar{v}_{\mathrm{s}}$ are more likely to produce a neutron in the primary interaction than $v \mathrm{~s}$, basically for all type of reactions. Thus the number of neutrons detected after a $v$ interaction is expected to follow this tendency. Despite of the fact that the neutrons produced in the final state increase with increasing energy because of interactions inside the nucleus, at energies particularly sensitive to $\mathrm{CP}$ violation effects (around $0.5 \mathrm{GeV}$ ) and Mass Hierarchy (few $\mathrm{GeV}$ ) their differences are significant. As illustration Fig. 2-top-left shows the multiplicities of tagged neutrons in $0.5 \mathrm{GeV}$ to $0.7 \mathrm{GeV} v$ and $\bar{v}$ interactions. The implementation of those, Gd driven, neutron multiplicity differences in the full oscillation analysis of atmospheric $v \mathrm{~s}$, will improve its sensitivity to the $v$ Mass Hierarchy by $\sim 50 \%$ and $\sim$ double that to $\delta_{C P}$ (Fig. 2-top-right).
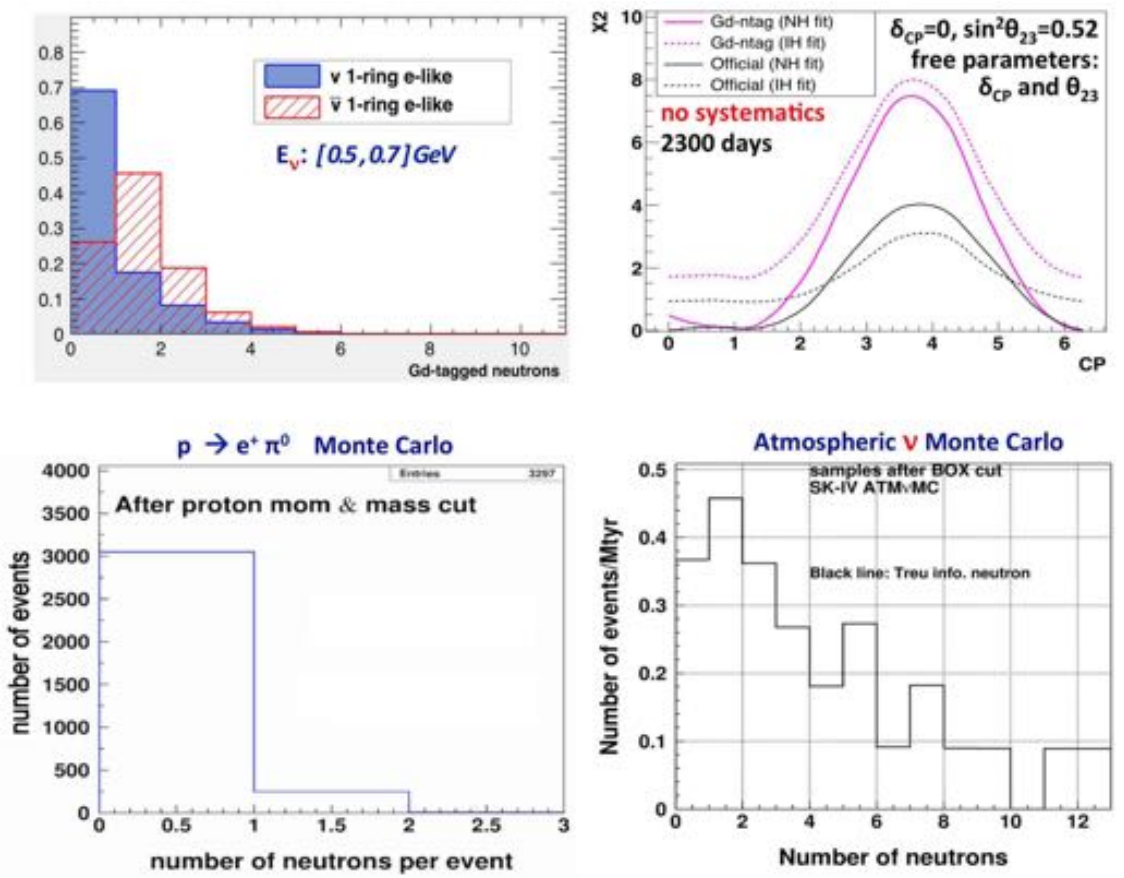

Figure 2: Impact of neutron tagging in high energy (order of $\mathrm{GeV}$ ) analyses. See text for details

In general, less neutrons should be emitted after genuine proton decays than in background processes caused by atmospheric $v$ interactions. Fig. 2-bottom-left shows the number of neutrons expected after proton decays via $p \rightarrow e^{+} \pi^{0}$. The requirement of no associated neutron to the events surviving the final selection cuts used in the current analysis, decreases the detection efficiency only by $6 \%$. On the other hand, the overwhelming majority of background events arising from atmospheric $v$ interactions have one or more neutrons in their final states (Fig. 2-bottom-right). The estimated reduction of the atmospheric $v$ background by neutron tagging (veto) with $\mathrm{Gd}$ is $\approx$ $83 \%$. Therefore a significant improvement on the sensitivity will be obtained. 


\section{The EGADS demonstrator}

The EGADS facility was built in the Kamioka mine explicitly to demonstrate that the gadolinium loading of Super-Kamiokande would be safe and effective. Its key elements are depicted in Fig. 3-left. They are a 200-ton water Cherenkov detector instrumented with the same 20-inch PMTs as those used in SK, a pre-treatment system with a 15-ton tank for dissolving and mixing $\mathrm{Gd}_{2}\left(\mathrm{SO}_{4}\right)_{3}$ into the water, the main $\mathrm{Gd}$-capable water circulation system, and a water transparency automatic measurement device (UDEAL).

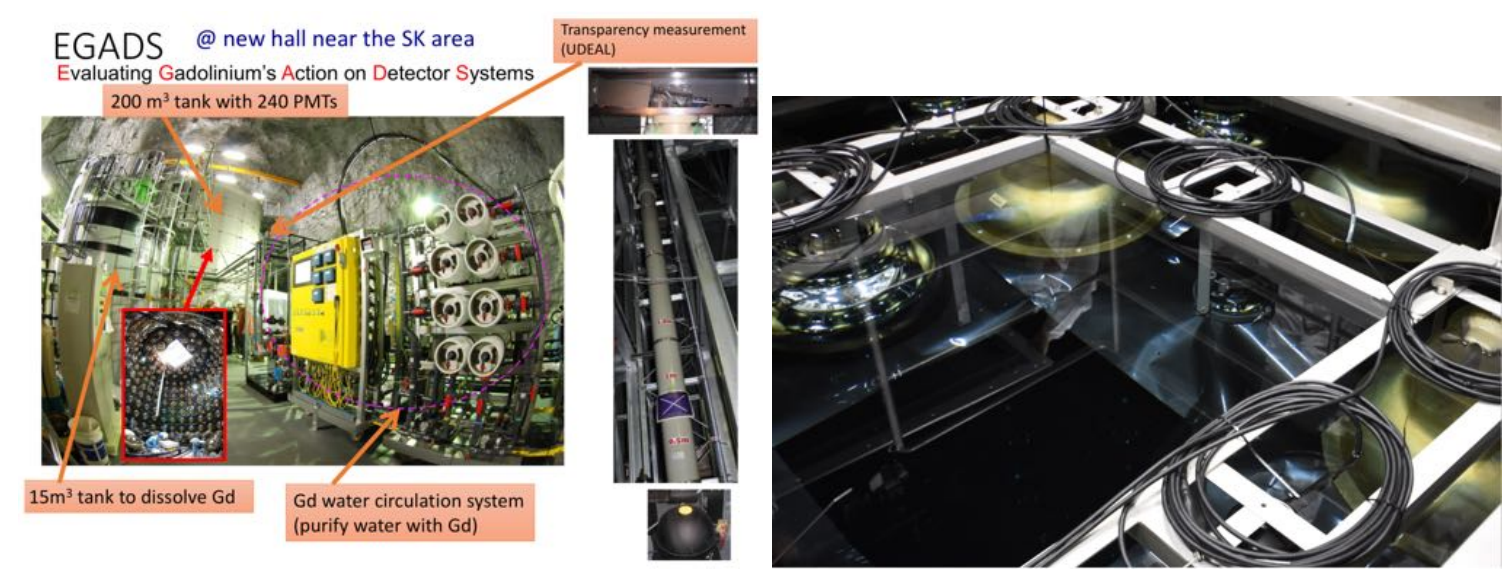

Figure 3: Left: a schematic view of the EGADS facility. Right: first look to the inside of the EGADS tank after two years of being fully loaded with Gd water.

The light attenuation length in water must be kept long to meet the various physics goals in Super-Kamiokande even if Gd is loaded. The main SK-Gd water system must be capable of performing selective filtration, since the desirable multivalent ions $\mathrm{Gd}^{3+}$ and $\left(\mathrm{SO}_{4}\right)^{2-}$ must be retained in solution while all other particles, gasses, and other contaminants must be removed. A new technology called "ionic band-pass filtration" has been developed for this very purpose [10].

The transparency of the water inside EGADS is monitored using the custom built UDEAL device (right-hand side of Fig. 3-left) that measures the absolute attenuation length at seven different wavelengths. Fig. 4-left shows the time evolution of the percentage of Cherenkov light that would be left after $15 \mathrm{~m}$ (the characteristic photon travel distance inside the SK detector) as calculated from a proper average of the seven wavelength UDEAL measurements. It demonstrates that 1) transparencies can be comparable to those attained at the pure-water SK's phases III and IV, 2) the period of recovery after any type of intervention ( $\mathrm{Gd}$ loading, water-system tuning, etc.) is rather short, and 3) in steady operation the measured transparencies are stable at values very appropriate for physics analyses.

The $\mathrm{Gd}_{2}\left(\mathrm{SO}_{4}\right)_{3}$ concentration in the 200-ton tank is monitored by using an Atomic Absorption Spectrometer (AAS). Water samples are collected at three different heights in the detector. The uncertainty of the measurement is around 3.5\%. Fig. 4-right shows the measured concentration since November $27^{\text {th }}, 2014$. The four loadings are clearly seen as steps in the concentration. The concentrations at all three sampling points are: 1) very close to each other, indicating that the solution is homogeneous within the tank, and 2) stable between loadings or any other external in- 
tervention, demonstrating that there is no significant Gd loss during continuous water recirculation and purification.
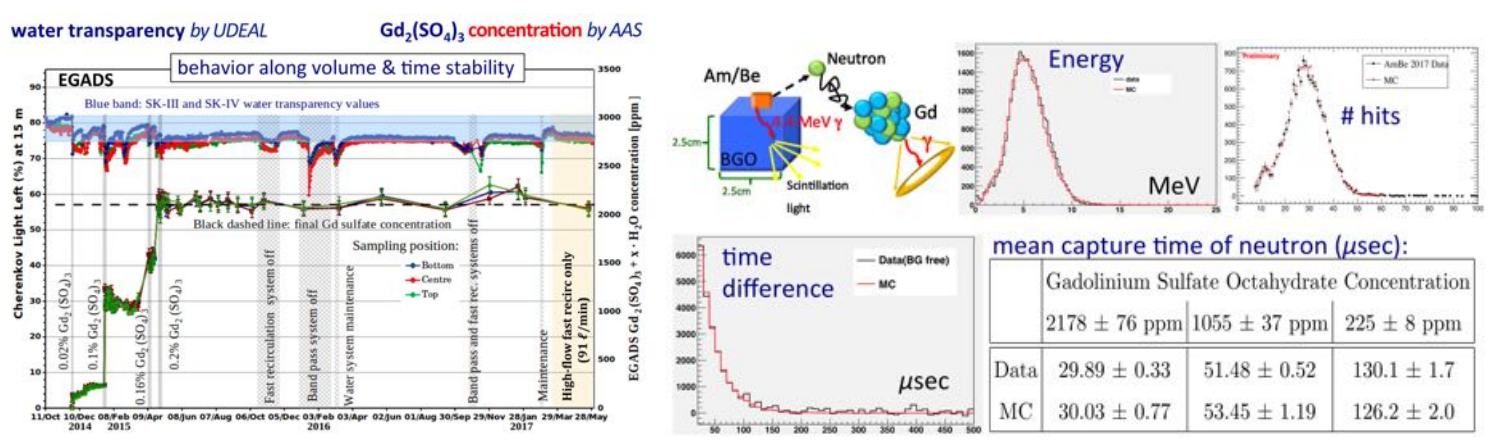

Figure 4: Evolution of light attenuation and Gd salt concentration with time (left). Demonstration of neutron tagging and quantification of its efficiency (middle and right plots). See text for details.

The demonstration of neutron capture by gadolinium-loaded water and evaluation of its efficiency is done using a Am/Be radioactive source, imbedded in a BGO crystal that is deployed inside the EGADS detector for the purpose. This system mimics the inverse $\beta$ signal (see Fig. 4left). The relevant reaction is ${ }^{241} \mathrm{Am} \rightarrow{ }^{237} \mathrm{~Np}+\alpha ; \alpha+{ }^{9} \mathrm{Be} \rightarrow{ }^{12} \mathrm{C}^{*}+\mathrm{n},{ }^{12} \mathrm{C}+\mathrm{n} ;{ }^{12} \mathrm{C}^{*} \rightarrow{ }^{12} \mathrm{C}$ $+\gamma(4.43 \mathrm{MeV})$ with the $\mathrm{BGO}$ emitting scintillation light when hit by $\gamma$-rays. Am/Be data were taken at most of the steps of $\mathrm{Gd}_{2}\left(\mathrm{SO}_{4}\right)_{3}$ loading i.e., at $0.02 \%, 0.1 \%$, and the full loading of $0.2 \%$. The lower-left panel of Fig. 4 shows the time difference from the prompt event to the neutron capture at $0.2 \%$ loading. Data and MC are in good agreement for the mean capture time in the three concentrations (see also panel bottom-right). The upper-middle plot shows the energy distributions of Gd $\gamma$-ray candidates, and the upper-right the detected number of hits, in both cases for data and $\mathrm{MC}$ at $0.2 \%$ loading showing the good agreement among them.

Figure 3-right shows a picture of the inside of the EGADS tank in May 2017 when it was opened for the first time after more than two years of being fully loaded with $0.2 \% \mathrm{Gd}_{2}\left(\mathrm{SO}_{4}\right)_{3}$ water. Everything looked clean and shinny: no sign of rust or deterioration of any type was apparent.

\section{Implementation in Super-Kamiokande}

Following a T2K+SK joint protocol, the starting of the SuperK-Gd project has been fixed to June $1^{s t}, 2018$. The overall plan of implementation is sketched in Fig.5-left. It has three phases. The first period is for refurbishment of SK and repair the small leak at the tank, pure water fill and recirculation of pure water in the detector until good water transparency is achieved $(\approx 6$ month total). The second period is for loading $\mathrm{Gd}_{2}\left(\mathrm{SO}_{4}\right)_{3}$ up to $0.02 \%$, which corresponds to $\approx 50 \%$ neutron capture efficiency on Gd. We will run the detector for a while at this concentration in order to study backgrounds and neutron capture efficiency. In the third period we will add the remaining $\mathrm{Gd}_{2}\left(\mathrm{SO}_{4}\right)_{3}$ needed to achieve our full target loading, $0.2 \%$.

Respecting the detector most critical items are the new water system and its refurbishment (mainly stop the leak). Respecting physics it is the radio-purity of the salt since whichever contamination it has, it will be distributed all along the fiducial volume of the experiment. 

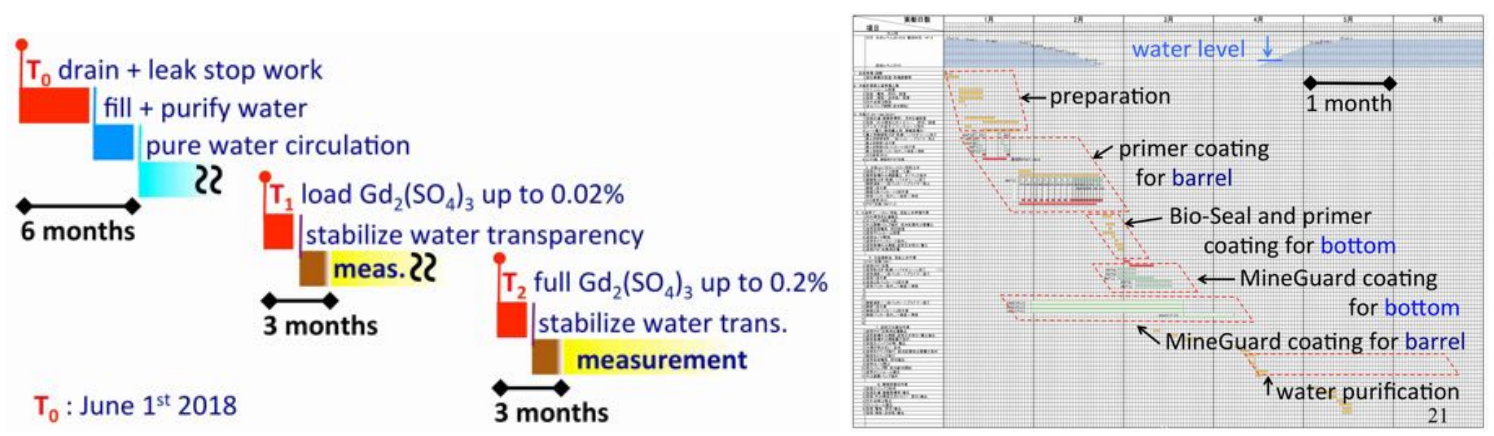

Figure 5: Left: plan for implementation of SuperK-Gd. Right: detailed schedule for refurbishment of the SK tank. See text for details.

The SuperK-Gd new water system, mainly a scaling up of EGADS, is designed as a highly compact, cost-saving system (Fig. 6-right). The system will be hosted in a new-built large experimental hall. The upper-left panel of Fig. 6 shows its location within the Kamioka mine. Lower-left and middle pictures show the inside of the hall and some details of the equipment already inside (mainly the pretreatment system). The hall has been constructed from the start to contain fluids and not release them into the mine. Finally, sump pumps will be in place to direct any spilled water through ion-exchange resin tanks.
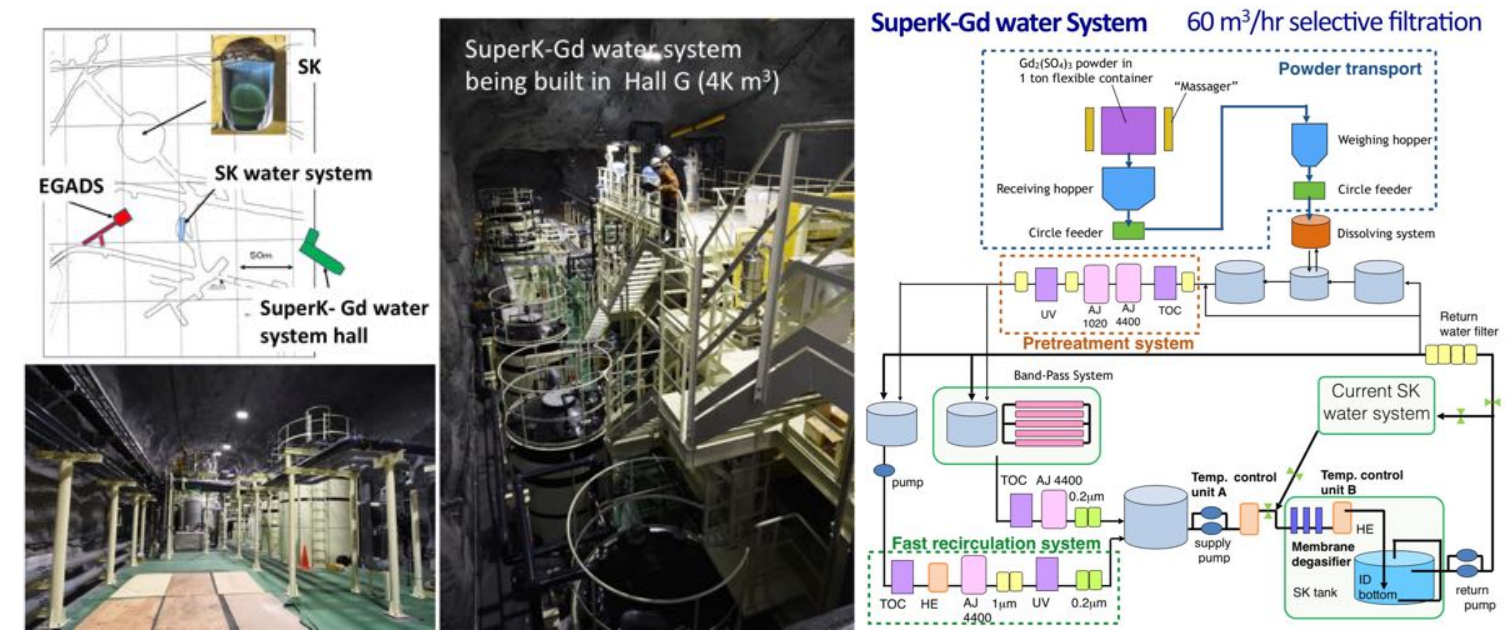

Figure 6: The SuperK-Gd water system complex. See text for details.

After detailed studies carried out to localize the leak/s we are rather confident that it is at the bottom of the tank. However the whole volume will be addressed in order to be in the safe side if energetic incidents occur (earthquakes etc.). All the submerged welded areas on the surface of the SK tank will be sealed using two sealants with very different mechanical properties. The first one, BIO-SEAL 197 (by Thin Film Technology, Inc.) is designed to sneak into small gaps and push entrained water out of the way. It is strong and rather rigid. The BIO-SEAL will be covered with MineGuard $\mathrm{C}$ that has no crack-seeking ability but possesses greater elasticity to allow for more displacement. Our MineGuard C uses poly-urea since it does not suffers from hydrolysis and shows very low radon emanation.

Adding the Gd salt to SK might jeopardize its scientific program if the salt is contaminated 
with radioactive isotopes. The left panel in Fig. 7-left summarizes the contaminations measured in many samples of $\mathrm{Gd}_{2}\left(\mathrm{SO}_{4}\right)_{3}$ from different companies. They are up to a few orders of magnitude larger than the required levels to guaranty reliable physics measurements; see the middle panel in Fig. 7 with those for SRN and low energy solar $v$ analyses. We are closely cooperating with several major companies specialized in high purity rear earth products. They are Molycorp, Shin-Etsu Che. Co. Ltd. , Kanto Che. Co. Inc., and Nippon Ytrium Co. Ltd.. As shown in the right-most panel of Fig. 7, some of them are able to provide samples that are already close to meet our specifications.

\begin{tabular}{|c|c|c|c|c|}
\hline $\begin{array}{l}\text { Typical activities of } \\
\text { (from over } 10 \text { samp }\end{array}$ & $\begin{array}{l}\text { salts in the marke } \\
\text { bles from } 5 \text { provide }\end{array}$ & & $\begin{array}{l}\text { Physics bas } \\
\text { radioactive }\end{array}$ & $\begin{array}{l}\text { ed requirements for } \\
\text { contaminations }\end{array}$ \\
\hline Radioactive chain & Part of the chain & $\mathrm{mBq} / \mathrm{kg}$ & $\mathrm{SRN}(\mathrm{mBq} / \mathrm{kg})$ & Solar $\nu(\mathrm{mBq} / \mathrm{kg})$ \\
\hline 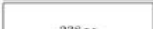 & ${ }^{238} \mathrm{U}$ & 50 & $<5$ & - \\
\hline${ }^{238} U$ & ${ }^{226} \mathrm{Ra}$ & 5 & - & $<0.5$ \\
\hline & ${ }^{228} R a$ & 10 & - & $<0.05$ \\
\hline${ }^{232} \mathrm{Th}$ & ${ }^{228} \mathrm{Th}$ & 100 & - & $<0.05$ \\
\hline $235 \mathrm{H}$ & ${ }^{235} \mathrm{U}$ & 32 & - & $<3$ \\
\hline & ${ }^{227} \mathrm{Ac} /{ }^{227} \mathrm{Th}$ & 300 & - & $<3$ \\
\hline
\end{tabular}

\begin{tabular}{|c|c|c|c|c|c|c|c|c|}
\hline Chain & \begin{tabular}{|l|} 
main \\
subchain \\
isotope
\end{tabular} & $\begin{array}{c}\text { GSF-1703- } \\
\quad C 9 \\
-702142 \\
\end{array}$ & $\begin{array}{l}\text { ICPMS } \\
\text { meas. }\end{array}$ & \begin{tabular}{|c|} 
GSF-1604- \\
C7 \\
-160303 \\
\end{tabular} & $\begin{array}{l}\text { ICPMS } \\
\text { meas. }\end{array}$ & $\begin{array}{c}\text { GSF- } \\
1611 \text { C8 } \\
-003\end{array}$ & \begin{tabular}{l|} 
GSF-1703 \\
C8 (RGD- \\
OSF-005)
\end{tabular} & $\begin{array}{l}\text { ICPMS } \\
\text { meas. }\end{array}$ \\
\hline \multirow{2}{*}{${ }^{238} \mathrm{U}$} & ${ }^{238} \mathrm{U}$ & $<13$ & 0.7 & $<20$ & 0.2 & $<13$ & $<9,2$ & 0.1 \\
\hline & ${ }^{226} \mathrm{Ra}$ & $0.7 \pm 0.4$ & & $<0.64$ & & $<0.3$ & $<0.26$ & \\
\hline \multirow{2}{*}{${ }^{232} \mathrm{Th}$} & ${ }^{228} \mathrm{Ra}$ & $<0.39$ & $23 T^{1.3}$ & $<0.67$ & ${ }^{212} \mathrm{Th}$ & $<0.3$ & $<0.26$ & ${ }^{22 x} T^{0.2}$ \\
\hline & ${ }^{228} \mathrm{Th}$ & $1.7 \pm 0.4$ & & $0.5 \pm 0.2$ & & $<0.4$ & $<0.37$ & \\
\hline \multirow{2}{*}{${ }^{235} \mathrm{U}$} & ${ }^{235} \mathrm{U}$ & $<1.3$ & & $<0.7$ & & $<0.6$ & $<0.51$ & \\
\hline & ${ }^{227} \mathrm{Ac} /{ }^{227} \mathrm{Th}$ & $<3.1$ & & $<2.3$ & & $<1.9$ & $<1.7$ & \\
\hline
\end{tabular}

Figure 7: About Radioactivity contaminations in SuperK-Gd. See main text for details.

All production batches of SuperK-Gd 100 tons of $\mathrm{Gd}_{2}\left(\mathrm{SO}_{4}\right)_{3}$ will be surveyed and those with excess contamination will not be allowed into SK. The work will be carried out at the Kamioka Observatory, the Boulby Underground Observatory and the Canfranc Underground Laboratory.

\section{References}

[1] Super-Kamiokande Collab.; Y. Fukuda et al., Phys. Rev. Lett. 81 (1989) 1562. Super-Kamiokande Collab. Y. Ashie et al., Phys. Rev. D71 (2005) 112005. Super-Kamiokande Collab.; S. Fukuda et al., Phys. Lett. B539 (2002) 179. Super-Kamiokande Collab.; K. Abe et al., Phys. Rev. D83 (2011) 052010. Super-Kamiokande Collab.; K. Abe et al., Phys. Rev. Lett. 110 (2013) 181802.

[2] K2K Collaboration, M.H. Ahn et al., Phys. Rev. D74 (2006) 072003. T2K Collaboration, K. Abe et al., Phys. Rev. Lett. 112 (2014) 061802 T2K Collaboration, K. Abe et al., arXiv:1707.01048v2

[3] Super-Kamiokande Collab.; K. Abe et al., Phys. Rev. D86 (2012) 012006. Super-Kamiokande Collab.; K. Abe et al., Phys. Rev. Lett. 113 (2014) 121802 Super-Kamiokande Collab.; K. Abe et al., Phys. Rev. D90 (2014) 072005.

[4] Super-Kamiokande Collab.; T. Tanaka et al., Astrophys. J. 742 (2011) 78. Super-Kamiokande Collab.; K. Bays et al., Phys. Rev. D85 (2012) 052007. Super-Kamiokande Collab.; H. Zhang et al., Astroparticle Phys. 60 (2014) 41.

[5] S. Horiuchi, J. F. Beacom, E. Dwek, Phys. Rev. D79 (2009) 083013.

[6] Super-Kamiokande Collab.; S. Fukuda et al., Nucl. Instr. Meth. A 501 (2003) 418. Nucl. Instr. Meth. A 737 (2014) 253.

[7] J. F. Beacom and M. R. Vagins, Phys. Rev. Lett. 93 (2004) 171101.

[8] Super-Kamiokande Coll., H. Watanabe et al., Astroparticle Phys. 31 (2009) 320.

[9] A. Odrzywolek, M. Misiaszek, M. Kutschera, Astroparticle Phys. 21 (2004) 303.

[10] M. R. Vagins (Tokyo U., IPMU), Nucl. Phys. Proc. Suppl. 229-232 (2012) 303. 